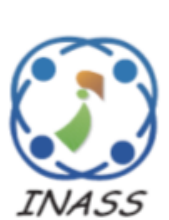

\title{
Retinal Image Lesions Assisted Diabetic Retinopathy Screening System Through Machine Learning
}

\author{
Yarragudi Madhu Sudhana Reddy ${ }^{1 *}$ \\ Ramaswami Sachidanandan Ernest Ravindran ${ }^{1}$ \\ ${ }^{I}$ Department of Electronics and Communication Engineering, Koneru Lakshmaiah Education Foundation, \\ Vaddeswaram, Guntur, Andhra Pradesh, India \\ *Corresponding author E-mail: ravindran.ernest@gmail.com
}

\begin{abstract}
This paper proposes an Automatic Screening System that identifies the symptoms of Diabetic Retinopathy from retinal fundus images and determines the severity. The entire system is of four-fold; the first phase pre-processes the retinal images and eliminates uneven illuminations. The second phase segments the major parts of images such as optic Disk and Blood Vasculature. Once the segmentation is completed, the residual part is called as lesion image and it is subjected to a hierarchical classification in the third phase. It is a two-step classification method which classifies the entire image into four regions namely Hard Exudates, Microaneursyms, Hemorrhages, cotton wool spots. For classification, three classifiers such as k-Nearest Neighbor, Gaussian Mixture Model and Support vector Machine are analyzed. Finally, the classified regions are processed through an objective function for severity grading. The novelty of this paper is a composite feature extraction method which extracts different and diverse features from lesion image. Since the lesions are very small and of different shapes, the proposed feature extraction method can provide more discriminant information for classifier. For experimental validation, a standard and publicly available DIARETDB1 dataset and the performance is measured through sensitivity, specificity, accuracy and area under curve. On an average, the proposed method gained accuracy, sensitivity, and specificity of $90.2 \%, 95.2 \%$ and $82.1 \%$ respectively.
\end{abstract}

Keywords: Diabetic retinopathy, Blood vessels, Optic disc, Lesions, Severity grading, Sensitivity, Accuracy.

\section{Introduction}

Recently, diabetic retinopathy (DR) is found as a serious chronic disease and conquered on third portion of diabetic patients over entire world. DR is the main reason of vision loss in adults of working age, and the prevalence of diabetes and the day by day count of diabetic patients are increasing rapidly in the world. According to the survey of American diabetes association (ADA), in the span of four years (2005-2008), the approximated number of people those were affected by DR is found to be 4.4 million of age 40 and below and it is approximated as $4.4 \%$ of overall diabetes patients. By 2040, it is predicted that the total number of people that will suffer from diabetes are approximately 642 million overall the world, while the DR effect one person from every three people [1]. Another survey ensures that the total number of people with DR will grow up to 191 million by the year ending of 2030 [2]. The detection of DR at its initial stages is slightly tough task but if it leaves as it is, it may consequences to severe DR followed by permanent vision loss. Hence, there is a need of regular checks ups which can help patients to delay and stop the progression of vision loss and blindness [3]. However, the scarcity of eye specialists results in a prolonged diagnosis and they can't cope up with the raise in the DR patients. So, developing an automatic DR detection system based on retinal fundus images is seriously required [4].

In retinal images, the major signs and characteristics of DR can are observed through hard exudates (HE), microaneursyms (MA), hemorrhages (HM), cotton wool spots (CWS) and nuerovascularization. The presence of one or all of these features in retinal images determines the DR status. At the initial phases, patients are observed without noticeable symptoms but in advance conditions, 
patients suffer from several kinds of symptoms like blurred vision, distortion and progressive visual severity loss. Based on these symptoms, the DR is graded into totally two grades, they are (1) Proliferative DR (PDR) and (2) Non-Proliferative DR (NPDR). Here the PDR indicates non DR while NPDR indicates the presence of DR. Further, the NPDR is sub-classified into three sub-classes; they are mild, moderate and severe. Mild is signified by small MA, moderate is reflected by the presence of HM and or HE. Severe NPDR is characterized by the rise in retinal ischemia through the appearance of small, abnormal and weak blood vessels which is called as Neuro-vascularization. The following figure. 1 shows the retinal images with different DR attributes.

HM looks like MA if it is smaller in size and MA looks like HM if it lies in wide blood vessel and larger in size. The physician can identify between these two signs by injecting fluorescein dye. At this situation, the HM won't change its color but MA turns into white color like the blood vessels. A one more solution is that the patient can pay for OCTA scan which was acquired by making the retina centered. The third sign in the pathology is due to the presence of HE. The HE's are the resultants of blood retinal barrier breakdown, allowing leakage of serum proteins and lipids from the blood vessels. Next, the main sign of PDR is neuro-vascularization which often occurs nearer to the Optic disk and hence it is called as NV of the disc (NVD). If the occurrence of $\mathrm{NV}$ is within three disc diameters of the major blood vessels, then it is called as NV elsewhere (NVE). These are the different possible ocular modalities those are generally used in the diagnosis of DR by ophthalmologists.

In the earlier days, the fluorescein angiography (FA) is generally used for the detection of retinal diseases. Next, fundus auto FA (FAF) has become

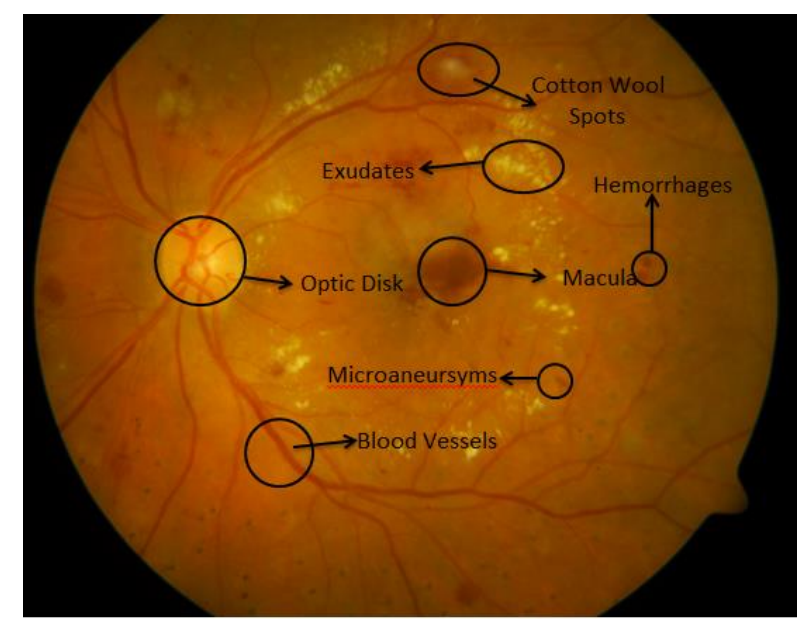

Figure.1. Retinal image with different DR attributes most commonly used method for pattern dystrophies and macular degeneration. By the year of 1990, optical coherence tomography (OCT) was discovered to visualize the layers of retina. The main intention of OCT is to detect choroidal NV and macular diseases. However, OCT is a subjective method and it is insensitive to the macular breaks and thin retinal vessels [5]. Recently, an extension of OCT called as OCT angiography (OCTA) is developed that mainly depends on the motion contrast of blood flow. Unlike FA, in OCTA, the dye is not injected and it is totally safe as well invasive. However, OCTA works only for the smaller field of view (FOVs) and it was not able to show the leakages. Based on these diagnosis methods, we understood that the colour fundus retinal image based DR diagnosis is most significant and widely applicable, particularly in the diagnosis of DR.

With this inspiration, we develop an automated DR severity grading system by analyzing the features of retinal images. The major contributions of this work are accomplished at four stages and they outlined as follows;

$>$ At first, we focus on the quality of retinal image and proposed new contrast enhancement method. This contribution normalizes the uneven illuminations and eliminates photographic artifacts in retinal images. This method enhances the contrast of image appropriately due to the consideration of mutual relationship between neighbor pixels. The earlier methods not focused in this direction.

$>$ The second contribution aims at the segmentation of major parts of retinal images; they are retinal vasculature and optic disc. As they are major portion of retinal images, the proper segmentation is required and it is done through the proposed method effectively. The proposed mechanism is effective than other methods because it adapts simple mathematical morphology and filtering techniques like median filter and Gaussian filter etc.

$>$ The third contribution aims at the classification of lesions such as HE, CWS, MA and HM. At this phase, a two-step hierarchical classification is developed. For this purpose, we proposed a novel feature extraction method which extracts different features including pixel intensity features and structural features. Due to different sizes, shapes and attributes of retinal lesions, a single feature cannot provide sufficient discrimination between them. Hence, we consider a set of different features (including structural ad pixel based features). The structural features provides shape and size information 
while pixel based features provide intensity, and illumination information. Most of the earlier methods employed only few features which can't contribute much for lesions identification.

$>$ The last contribution aims at the grading of DR severity based on the classified results in third stage. As the lesions are more important for DR severity grading, consider to grade the DR based on the obtained lesions.

Rest of the paper is structured as follows; the details about earlier DR grading systems are explored in section 2. Section 3 explores the complete particulars of developed DR grading mechanism in four stages. Section 4 illustrates the details of experimental validations and results observations. Finally, the concluding remarks are exposed in section 5 .

\section{Literature survey}

So many researches have proposed concentrated in the diagnosis of DR through retinal images and they developed different methods. For example, Brain et al. [6] used HM and HE for the diagnosis of DR. Initially, they focused on the contrast enhancement and they applied contrast limited adaptive histogram equalization (CLAHE) [7]. Then they segmented OD region from enhanced green plane of image. Afterwards, they applied a stack of gabor filters followed by thresholding for the extraction of exudates. Next, the HM is extracted through the circular hough transform (CHT) followed by thresholding. However, the authors segmented only the signs of exudates and HM without grading the grade of DR. Moreover, CLAHE is not effective for contrast enhancement.

Atlas and parasuraman [8] applied three feature extraction methods namely gray level co-occurrence matrix (GLCM) [10], speed up robust features (SURF) [11] and gary level run length matrix (GLRLM) [9] for the classification of normal retinal images from DR effected retinal images. For classification purpose, they adapted for an adaptive neuro-fuzzy inference system (ANFIS) and extracted HM. However, HM alone is not sufficient to grade the DR from retinal images.

Orlando et al. [12] used support vector machine algorithm for the extraction of blood vessels in retinal images. With respect to the pixel distances, they weighed the pairwise interactions. They also used 2D gabor filter and unary potentials of line detector for the purpose of image standardization. But, the authors neglected the advantages of automated segmentation that have significant impact in the negative results. Moreover, the line detectors are not able to segment lesion because they are not linear structures.

Fadafen et al. [13] applied mathematical morphology over retinal images after the exclusion of OD and they tried to extract exudates. They used feature and edge based identification of blood vessels through three features namely direction, width and brightness. However, the results derived are totally dependent on the nature of human visual system (HVS) which is more sensitive to directions and intensities of pixels. Even though exudates are treated as a strong reference or sign for the diagnosis of DR, the authors are not able to classify the grades of DR. Furthermore, they didn't use any contrast enhancement method and hence the obtained results are not satisfactory.

D. W. Safitri and Juniati [14] aimed at the diagnosis of normal from DR. Initially, they enhanced the low contrast retinal image through CLAHE. Then they focused on the blood vasculature segmentation through matched filter followed by thresholding. Finally, they used the box-counting method [15] for the fractal dimension and k-nearest neighbor (k-NN) algorithm for classification. However, the results are totally dependent on the dimensions of fractal values. Hence, the obtained performance is not sufficient for ML imbalanced dataset.

Abdelmaksoud et al. [16] considered four attributes namely HE, MA, HM and blood vasculature $(\mathrm{BV})$ for the diagnosis of $\mathrm{DR}$ grades through retinal images. They employed three methods namely $1^{\text {st }}$ order Gaussian derivative filter, matched filter and mathematical morphology. They extracted BV counts, lesions area, and GLCM. For classification purpose, they adapted SVM algorithm. GLCM is determined through pixel correlation but it is not sufficient to extract the lesions which have similar pixel intensities but different sizes and shapes.

Recently, due to the widespread advancement in the deep learning, some authors employed several deep learning algorithms for the diagnosis of DR grades through retinal images. The main advantage with deep learning methods is their efficiency in lessening the effort at feature extraction. M. D. Abramoff et al. [17] analyzed the software of the IDx-DR device [18]. This device considers OD and macula centered images for every eye as input and produces the DR grade as an output. The main sources of this device are Oxford visual geometry and AlexNet [19]. The authors altered the system of device such that it can be applied on the public datasets. However, it is not able to detect the PDR from macular edema (ME) separately. Furthermore, 
the drift in the diagnosis of MA and HM affected the detection of moderate and mild DR grades.

Bellemo et al. [20] developed an integrated CNN model by combining two CNN models. They are a residual neural network (ResNet) [21] and an adapted visual geometry group network (VGGNet) [22]. The authors employed gradient descent method for the classification of retinal images. The classification probabilities of two models are summed up and they are used as a final classification result to measure the specific and sensitivity scores. Though they gained a superior recognition performance, they suffered with larger computational time. Moreover, the ResNet and VGGNet is complex in structure and create huge processing time even for classification of smaller sized retinal images.

Unlike the above methods, mansour [23] applied AlexNet for the extraction of blood vessel features. They utilized the theory of connected component analysis (CCA) [24] for feature extraction followed by feature selection. The AlexNet is a seven layer network which consists of five convolutional layers and two fully connected layers. Finally, they employed SVM algorithm for classification of DR grades. However, the BV explores the details of severe stage of DR but it can't support for other grades.

Gadekallu et al. [25] applied principal component analysis (PCA) [26] and reduced the dimensions by firefly. The authors' normalized through standard scalar method. Their model was being over fitted during the implementation on smaller sized datasets.

Hagos et al. [27] used a pre-trained deep learning model called as Inception-V3 [28] to classify the DR into two grades, they are normal and abnormal. Initially, the retinal image is pre-processed by cropping and resizing images. They used softmax classifier and stochastic gradient descent (SGD) for optimization. The authors just classified the absence and presence of DR while it is not sufficient for real time diagnosis. Since there exists different levels of $\mathrm{DR}$, the classification needs to be fine-tuned.

Similarly, Tymchenko et al. [29] also used deep learning model called as EfficinetNet based on the standard pre-trained model ImageNet [30]. They initially pre-processed the retinal image through rotation, transposing and vertical flipping. Though they used more than one dataset for evaluation, this model is not sufficient for the purposes of real time applicability. Hence they decided to use shapley additive explanation (SHAP) [31] in future for feature visualization that helps a physician in the assessment of DR grades.

$\mathrm{Xu}$ et al. [32] also concentrated on the detection of presence or absence of DR. For this purpose, they presented a CNN model with ten layers. After every 2 convolution layers, one max pooling layer is inserted followed by fully connected layer. They used softmax classifier and SGD optimizer and cared about the image pre-processing by subjecting it to augmentation. However, they tested only on a small sized dataset which is not sufficient for real time applications.

Pratt et al. [33] modeled a 13-layered CNN model among which ten are convolutional layers and three are fully connected layers. They aimed at the determination of five classes and employed softmax classifier. Moreover, they used rectified linear unit (ReLU) as an activation function. However, they ignored image pre-processing stage and hence the results are observed as unsatisfactory for noisy images. Moreover, it is mandatory to employ more than one dataset such that the robustness will get proved.

From the above mentioned methods, we found that no method is there which construct a complete DR diagnosis system. Some methods considered only few attributes which are not sufficient for the diagnosis of DR. Some method applied advanced method like deep learning for DR diagnosis. However, they constitute a huge computational burden over the system and gained only an acceptable performance. Different from these methods, in this paper, we construct a complete DR diagnosis system that considers all the attributes along with simple machine learning algorithms. Due to the simple algorithms, the proposed method is observed to have less computational complexity.

\section{Proposed approach}

\subsection{Overview}

In this section, we explore the complete details of proposed DR diagnosis system. The proposed system totally consists of four phases; they are (1) preprocessing, (2) segmentation or background masking, (3) lesions classification and (4) severity grading. In the first phase, the input retinal image is subjected to pre-processing, means the external effects such as nose and uneven contrast illuminations and eliminated. In the second phase, this work aimed at the segmentation and masking of retinal vasculature and optic disk which are major parts of retinal image. These two regions are treated as background regions since the lesions are treated as foreground objects here. For the segmentation of retinal vasculature and optic disk, we applied two different mechanisms. Once the Retinal vasculature and optic disk are 


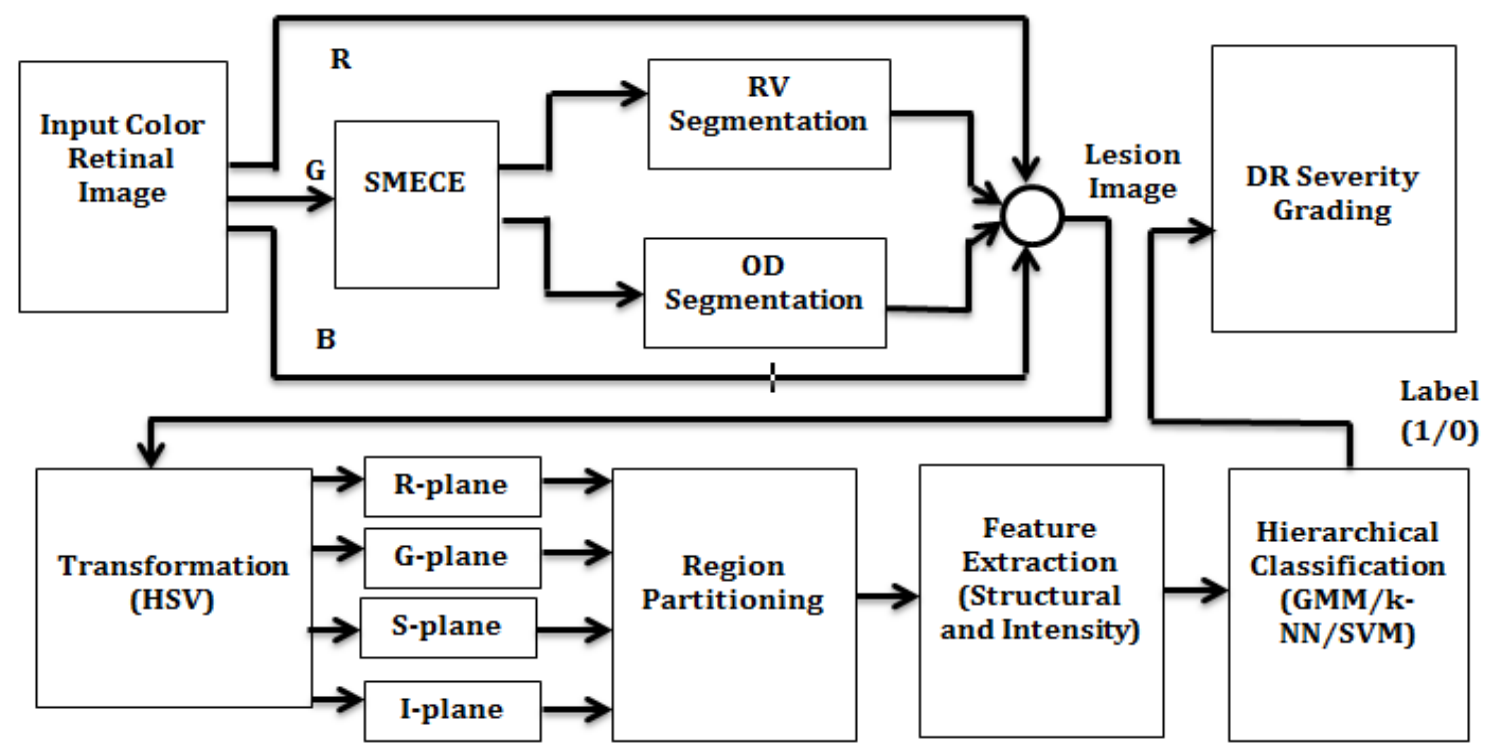

Figure. 2 Functional block diagram of proposed system

segmented, they are masked out and resulted in an image with only lesions or foreground objects. In the third phase, the lesion image is subjected to hierarchical classification because there exists two types of lesions and sub-lesions in each lesion category. For lesions classification, the lesion image is initially partitioned into several regions through spatial windowing and then each region is processed for feature extraction followed by classification. At classification, we employed different machine learning algorithms such that we can understand the robustness of developed system. At the last phase, the classified regions are used for grading the severity of DR based on a combination function. The overall functional block diagram of proposed approach is shown in Fig. 2.

\subsection{Pre-processing}

In the pre-processing phase, the retinal image is pre-processed to eliminate the false photographic artifacts and uneven illuminations. For contrast enhancement, the green plane of retinal image is fed as input and enhanced through spatial mutual entropy based contrast enhancement (SMECE) [34] which considers the spatial relationships between image pixels. For a given input image, the SMECE initially partitions it into several overlapping blocks which are called as spatial grids. After partitioning, each grid is subjected to histogram computation followed by entropy calculation. Then the histograms of each grid are normalized through grid size and then summed up such that the sum is equal to 1 . Based on the normalized histograms, the spatial mutual entropy (SME) is measured between different histograms in the same grid. Consider $h_{k}$ is the normalized histogram of $k^{\text {th }}$ gray level in a particular spatial grid and $h_{l}$ be the normalized histogram of $l^{\text {th }}$ gray level in the same spatial grid, the SME is measured by finding the joint histograms $\left(h_{k, l}\right)$ as

$$
h_{k, l}=\min \left(h_{k}, h_{l}\right)
$$

Based on the above joint histogram, a new relation called as Mutual Relation is derived for every two distinct gray levels. The Mutual Relation is measured as

$$
M R_{k, l}=\sum_{h=0}^{H-1} \sum_{w=0}^{W-1} h_{k, l}(h, w)\left(\frac{h_{k, l}(h, w)}{h_{k}(h, w) h_{l}(h, w)}\right)
$$

Where $h$ and $w$ denote the length and width of region respectively. $h_{k}(h, w)$ and $h_{l}(h, w)$ are the normalized histograms of $k^{\text {th }}$ and $l^{\text {th }}$ gray levels respectively in the spatial grid of size $h$ and $w$. The MR is observed to be high when the occurrence of two gray levels is very close in the spatial grid. In such scenario, the gap must be high between adjacent gray levels. Here, the contras enhancement is done by mapping input gray levels with output gray levels and during the mapping, the gray levels are adjusted such that the output pixel contrast must be different with the contrast of input pixel. After the determination of MR between gray levels, one rank is assigned for every relation and the gap is adjusted based on the rank. This gap is multiplied with input gray levels and the result is output gray level.

\subsection{Segmentation}

In this phase, the contrast enhanced image is fed as input and it was segmented of masking. Here the 
main aim of segmentation is to partition the two background regions; they are retinal vasculature and optic disk. In this work, the lesions are treated as foreground objects while retinal vasculature and optic disk are considered as background regions. For segmentation of two regions, we adopted two different methods as they are two different objects with different properties. The details of methodologies employed for segmentation are explored in the following sub-sections;

\subsubsection{Retinal vasculature segmentation}

The RV segmentation is done in two steps; they are (1) major and minor blood vessel segmentation, (2) vessel pixel and non-vessel pixel identification followed by post-processing [35]. Since the major vessel structure is much differed from minor vessel structure, we adopted different statistics for their segmentation. Initially, the contrast enhanced green plane of retinal image is for major vessels extraction. Next, for minor vessels extraction, the negative of blood vessel image is processed. Finally, to get the overall vascular structure, the vessel structures obtained in the above two phases are fused. At the segmentation of major blood vessel structure, the retinal image is initially passed through a low pass filter (LPF) and the output image is subtracted from input image. Then the resultant image is subjected to thresholding process to determine the pixels those values less than zero. Next, for the obtained output, the absolute pixel strengths are calculated and adjusted appropriately such that they will look clearer. For the extraction of minor vessels, the negative of vessel enhanced image is processed. The input is convolved with different gabor filter kernels at different orientations. The orientational difference is set as $15^{\circ}$ and hence the total number of orientations possible is 12 . At every orientation, the input image is convolved with a Gabor kernel. Hence the total number of filtered responses for each pixel is 12 . Among the 12 filtered responses, the presence of minor vessels is determined by finding the maximum response value. Since the minor vessels are very low intensive and negligible, the identification is slightly tough task. For this purpose only, we have employed gabor filter which is a robust orientational filter that alleviates the presence of minor vessels. For each pixel, the obtained maximum response value designates its presence in that particular orientation. After the completion of minor vessels, the major blood vessel image is intersected with minor blood vessel image to form complete vascular structure.

To further polish the vessel structure, an identification method is employed which identifies vessel pixels from non-vessel pixels. This identification process is done over the vessel subimage which left after the extraction of complete vascular structure in first step. For this identification purpose, the vessel sub-image is subjected to feature extraction followed by classification. For feature extraction purpose, three different methods are used; they are gradient method, correlation method and intensity method. These methods determine three different features and then a composite feature vector is formed by their integration. The intensity method fetches intensity features such as mean, standard deviation, maximum and minimum. Next, for gradient features extraction, frangi filter [36] is applied by deriving $2^{\text {nd }}$ order partial derivatives of Hessian matrix followed by Eigen values computation. Thirdly, for correlation features, the linear correlation is measured between image blocks at different pixel centers. After the completion of feature extraction, they fed to SVM classifier for the classification of vessel pixels from non-vessel pixels. Here, the SVM classifier assigns class label 1 for vessel pixels and class label 0 for non-vessel pixels. Finally, the vessel pixels are added to the output vessel structure obtained in the first step and it is called as post-processing.

\subsubsection{OD segmentation}

OD is one more major part of retinal image which was mostly used in the diagnosis of DR and also several eye related diseases. The OD segmentation is done in two steps, (1) OPD locating and (2) OD determination [37]. For OD determination, an edge density filter (EDF) is applied which determines the complete pixels of OD based on connected opponents. Initially, the retinal image is processed for locating center of OD called as OD pixel (ODP). For this purpose, the input green plane of retinal image is passed through three different filters namely median filter, variance filter and Gaussian filter. After getting the filtered response of each filter, the approximate ODP candidate locations are measured by the computation of maximum value in each response. The filter size of median filter and variance filter are set as $21 \times 21$ and $51 \times 51$ respectively. After the determination of approximate ODP candidate locations, the centroid is measured by averaging them in each filtered response. Now, based on the centroid, the exact ODP location is finalized based on two aspects, they are (1) if the three candidate locations are nearer to centroid, then the centroid itself becomes ODP. (2) If any two candidate locations are nearer to centroid, then the ODP location is an average of those two candidate locations. 
Once the ODP is located, a sub-image of size $400 \times 400$ is cropped along both row side and column side and it is called as OD sub-image. Then the OD sub-image is subjected to edge detection and binarization through Otsu thresholding [38]. For edge detection, the canny edge detector is applied over OD sub-image and the size of filter is set as $3 \times 3$. Over the obtained canny edge response, the gradient magnitudes are measured with modulo of partial derivatives of every pixel. Next, for binarization, we adopted for Otsu thresholding and the pixel whose magnitude is greater than the threshold is designated as 1 otherwise 0 . Now, the resultant image is a binary image which consists of only 1's and 0's. In this image, almost the 1's represents bright regions and 0 's represents non-bright regions. Here, the OD pixels are having 1 and non-OD pixels are having 0 . From the binary image, the OD region is obtained by the determination of connected components with the help of EDF. The EDF connects the regions with high edge density and removes other regions. Since the OD is a brighter region, almost all the pixels lies in it are designated by 1 and connecting all those pixels constitutes the OD region. In this manner, the OD is segmented and masked out from the retinal image.

\subsection{Lesions classification}

Once the image segmentation is completed, the residual image consists of only foreground objects corresponding to bright lesions and red lesions. The classification of these lesions is further required to lessen the false positivity. Here, the bright lesions are classified as CWS and HE while the red lesions are classified as MA and HM. Towards this two-step classification, the lesion image (image remained after the segmentation of retinal vessels and optic disk) is subjected to spatial windowing, feature extraction and classification. During the training process, the features of each region are scaled in the range of 0 and 1 . If the region is manually annotated as lesion, then the class label $y$ is assigned as 1 otherwise the class label $y$ is assigned as 0 . This is validated with the help of ground truth and the regions those were not matched are assigned to class label $y=0$. During the testing process, the feature vectors of regions are similarly scaled in the range of 0 and 1 . Next, they are subjected to testing and their class label is determined through ground truth are compared with the class labels obtained through classifiers which corresponds to class 0 or 1 . Here, totally three classifiers are used to analyze the performance; they are k-nearest neighbor (k-NN), Gaussian mixture model (GMM) and support vector machine (SVM). The first two classifiers are low complex classifiers and the last one is of heavier complexity. All are individually analyzed over the extracted features of lesions image. The SVM classifier is realized through MATLAB software with the help of libSVM toolbox.

\subsubsection{Spatial windowing}

For the extraction of features of every object, initially the lesion image is partitioned into several overlapping regions. For this purpose, we apply spatial windowing technique with different sizes of window. For windowing, every pixel of image is centered and region is extracted based on the size of window. Consider $I_{l}$ be the lesion image, the window with a pixel located at position $(x, y)$ is denoted as $I_{l}^{w}(x, y)$. The spatial windowing is done in totally four different versions of lesion image; they are green plane $\left(I_{G}\right)$, red plane $\left(I_{R}\right)$, saturation plane $\left(I_{S}\right)$ and intensity plane $\left(I_{I}\right)$. Since our main intention is to identify both bright and red lesions, we had done spatial windowing in four planes. Each plane has its own significance in the provision of clear information about the properties of bright lesions and red lesions. The bright lesions are easily are easily identified in saturation and intensity planes while the remaining two planes (green and red) contributes to the red lesions. The properties of red lesions mostly resemble with vessel pixels properties at green and red planes. Hence the first two planes are adapted for red lesions. Next, the bright lesions are characterized by intensities, as they are brighter than red lesions. Such kind of information is available only through saturation and intensity planes. Hence they are adapted for the bright lesions.

\subsubsection{Feature extraction}

Feature extraction is very important is lesions classification. Some prior works are already introduced several features for lesions classification. For instance, [39] extracted totally 13 features for lesions classification with the help of spencer-frame system and later it was extended to 68 features [40]. Moreover, some more works are also extracted several features for lesions classification. Inspiring from these works, in this paper, we extracted totally 30 features that are mostly taken from previous works along with some extra features. Among these 30 features, 14 features are structural features and the remaining 16 are pixel based features. Some examples of structural features include area, solidity, and eccentricity etc. while mean, standard deviation, maximum and minimum are pixel intensity based features. The details 30 features are explored here very clearly. 


\section{Structural features}

1. Area: Area is measured by the computation of total number of pixels in the region.

2. Minimum Distance from OD region: This is measured as the distance between the center pixel in OD region and the center pixel in the current region.

3. Length of Major Axis: This is measured as the length of major axis of ellipse surrounded over the region

4. Length of Minor Axis: This is measured as the length of minor axis of ellipse surrounded over the region

5. Diameter: The region bounded with a circle and its diameter is considered as this feature.

6. Rectangle Length: The region is bounded by a bounded box and its length is considered as rectangle length. The rectangle size must be chosen in such a way, it must be small and covers the entire region.

7. Eccentricity: This feature is measured for an ellipse estimated around a region.

8. Filled Area: This feature is measured as the area of region after filling it with holes.

9. Rectangle Width: The region is bounded by a bounded box and its width is considered as rectangle width.

10. Minimum Distance from Retinal Vasculature: This is measured as the distance between the center pixel in retinal vessel region and the center pixel in the current region.

11. Orientation: this feature is measured as an angle between $\mathrm{x}$-axis and the major axis of ellipse.

12. Perimeter: This feature is measured as a distance around the object region.

13. Solidity: this feature is measured as the ratio of area to the convex area.

14. Euler Number: This feature the Euler number of a region.

\section{Pixel based features}

Under this set of features, we extracted totally 16 features and they are broad categorized into four categories, they are maximum, minimum, mean and variance. Since the input image is of four forms (green, red, saturation and intensity planes), the above four types of features are extracted from every image model, the total number of features are $4 * 4=$ 16. Consider $v_{i=\{G, R, S, I\}}$ be the variance features of $i^{\text {th }}$ form, it is measured as

$$
v_{i=\{G, R, S, I\}}=\text { variance }\left\{I_{l}^{w}(x, y)\right\}^{i}
$$

Where $i$ varies for image models, $w$ is window, $l$ is lesion and $(x, y)$ denotes the pixel position of center pixel. Similarly, the remaining features such as mean, maximum and minimum are computed as

$$
\begin{aligned}
& \mu_{i=\{G, R, S, I\}}=\operatorname{mean}\left\{I_{l}^{w}(x, y)\right\}^{i} \\
& M x_{i=\{G, R, S, I\}}=\operatorname{maximum}\left\{I_{l}^{w}(x, y)\right\}^{i} \\
& M n_{i=\{G, R, S, I\}}=\operatorname{minimum}\left\{I_{l}^{w}(x, y)\right\}^{i}
\end{aligned}
$$

So, from the Eqs. (3-6), each region is represented with 16 intensity features among which first four are variance, next four are mean, next four are maximum and last four are minimum features. Among every four features, the first one is extracted from green plane, second one is extracted from red plane, third one is extracted from saturation plane and the last one is extracted from intensity plane. In this way, every region is represented with 16 pixel intensity based features.

Finally, each region is represented with 30 features which explore almost all kind of information in the retinal image about lesions. Among these 30 features, the 14 structural features are used initially for the classification of lesion regions from nonlesion regions. For instance, the CWS and HE have larger distance from OD region when compared with the non-lesion regions. Next, for the classification of red lesions, we consider that the non-lesion regions represent fine blood vessels and hence they are located at smaller distances from blood vessels when compared with the red lesions. Moreover, due to the elongated structures of retinal vessels, the ratio of major axis to minor axis length is higher for nonlesion regions than the red- lesions. In the second step of classification, the brightness of $\mathrm{HE}$ is much compact than the CWS and also have well defined boundaries. On the other hand, the CWS are larger in area and less compact in nature. This compactness is assessed through the structural feature called as solidity.

\subsubsection{Classification}

Under the classification, we accomplished a twostep hierarchical classification. In the first step, the bright lesion regions are classified as true bright lesion regions $\left(R_{T B}\right)$ and false bright lesion regions $\left(R_{F B}\right)$ or non-lesions regions. In this classification, the $R_{T B}$ is assigned to the class label of 1 and $R_{F B}$ is assigned to the class label of 0 . Next, the red lesion regions are classified as true red lesion regions $\left(R_{T R}\right)$ and false red lesion regions $\left(R_{F R}\right)$ or non-lesions regions. In this classification, the $R_{T R}$ is assigned to the class label of 1 and $R_{F R}$ is assigned to the class label of 0 . In the second step of classification, the true bright lesion regions are classified as HE regions 
$\left(R_{T B}^{H E}\right)$ and CWS regions $\left(R_{T B}^{C W S}\right)$. In this classification, the $R_{T B}^{H E}$ is assigned to the class label of 1 and $R_{T B}^{C W S}$ is assigned to the class label of 0 . Next, the true red lesion regions are classified as MA regions $\left(R_{T R}^{M A}\right)$ and $\mathrm{HM}$ regions $\left(R_{T R}^{H M}\right)$. In this classification, the $R_{T R}^{M A}$ is assigned to the class label of 1 and $R_{T R}^{H M}$ is assigned to the class label of 0 . In this way, the entire lesions are classified into four categories and they are used in grading the severity of DR.

\subsection{Severity grading}

After the completion of lesions classification, then they need to be fused to find out the severity or level of DR which is called as severity grading. The result of classification is that every region is assigned to one label, i.e., HE, CWS, MA and HM. Now all these regions are need to be fused. Before fusing them, the total numbers of similar regions are accumulated in each image. The accumulation is done as follows;

$$
\begin{aligned}
& H E=\cup_{k} R_{H E}(k) \\
& C W S=\cup_{k} R_{C W S}(k) \\
& M A=\cup_{m} R_{M A}(m) \\
& H M=\cup_{m} R_{H M}(m)
\end{aligned}
$$

Where HE, CWS, MA and HM represents the total number of regions belongs to hard exudates, cotton wool spots, microanersyms and hemorrhages respectively. Now the severity grading of DR is done through a combination function which combines HE, CWS, MA and HM.

$$
\text { Grade }(G)=\varphi(H E, C W S, M A, H M)
$$

Where $G=\{0,1,12,3\}$ which implies four possible grades. An image with $\mathrm{G}=0$ implies no $\mathrm{DR}, \mathrm{G}=1$ implies mild DR, $G=2$ implies moderate $D R$ and $G$ $=3$ implies severe DR. The combination function $\varphi$ is specified in the table. 1 for MESSIDOR dataset as

The effect of lesion combination is studied in [41] to determine the grade of DR. These works demonstrate that clinically relevant lesion combination needs more accurate estimation

Table 1. Combination function of MESSIDOR dataset

\begin{tabular}{|c|c|}
\hline Grade & Combination \\
\hline 0 & $\mathrm{MA}=0$ and $\mathrm{HM}=0$ \\
\hline 1 & $(0<M A<5) \&(H M=0)$ \\
\hline 2 & $(5<M A<15) \&(0<H M<5)$ \\
\hline 3 & $(M A \geq 15) \&(H M \geq 5)$ \\
\hline
\end{tabular}

regarding the number of red lesions than bright lesions. In case of bright lesions, eh raise in false positivity may imply macular degeneration or retinal abnormalities other than NPDR. Hence, detection of bright lesions results in less FP. But for red lesions detection, the wrong result implies the false interpretation of DR severity. Hence, it is necessary for any automated system to detect both bright and red lesions more accurately.

\section{Simulation results}

This section explores the details of simulation experiments. Initially, we discuss about the details of datasets are employed. Next, the details of performance metrics are explored. Finally, the results obtained after the deployment of proposed mechanism over specified datasets are explored. For simulation purpose, we used MATLAB software. The simulation is done on a computer with i5/2.4 GHz processor with 8 GB RAM and an NVDIA VGA card.

\subsection{Dataset}

DIARETDB1: For the validation of proposed approach, we used DIARETDB1 dataset which consist of totally 89 retinal images. According to the experts suggestion, there exists only five images are normal and the remaining are at least mild NPDR. The dataset has ground truth images for all the retinal images in MA, HM and soft exudates and HE signs. All the images are acquired with the help of same $50^{\circ}$ field of view digital fundus camera with different settings. This dataset is referred to as "calibration level 1' fundus images. AN example retinal image and the corresponding ground truth results are shown in Fig. 4.
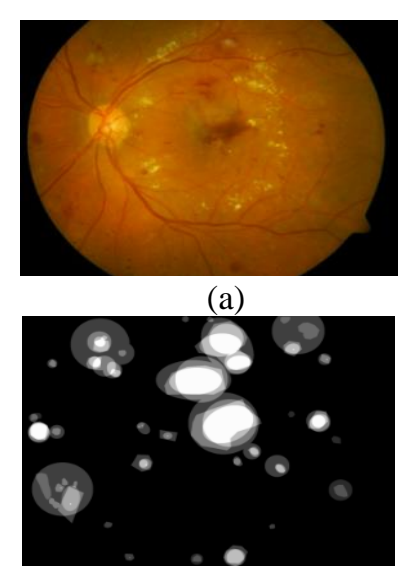

(c)

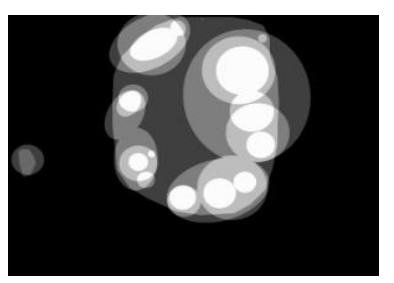

(b)

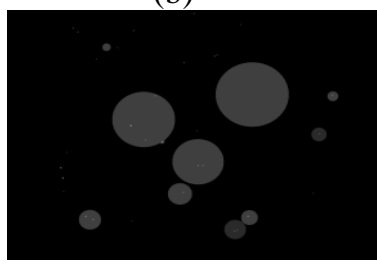

(d)
Figure. 4 (a) Original retinal image, (b) hard esudates, (c) hemorrhages, and (d) red small dots 


\subsection{Performance metrics}

Under this section, we have considered several performance metrics for the evaluation of proposed method's performance. They are Sensitivity (Sen), Specificity (Spe), Area Under Curve (AUC), and Accuracy (Acc). The mathematical representation of these metrics is depicted below;

$$
\begin{aligned}
& \text { Sensitivity }=\frac{T P}{T P+F N} \times 100 \\
& \text { Specificity }=\frac{T N}{T N+F P} \times 100 \\
& \text { Accuracy }=\frac{T P+T N}{T P+T N+F P+F N} \times 100 \\
& A U C=\frac{1}{2}(\text { Sensitivity }+ \text { Specificity })
\end{aligned}
$$

Where TP is true positive, TN is true negative, FP is false positive and FN is false negative. After the segmentation, these are measured immediately and then they are substituted in the above equations. The meaning of these metrics changes according to the classification. In the first step of classification, if the lesion regions are classified as lesions, then they are considered as TPs otherwise false negatives. In the second step of classification, particularly for bright lesions, if the region with HE are classified as same, then they are considered as TP otherwise FN. On the other hand, for red lesions, if the regions with MA are classified as same, then they are considered as TP otherwise FN. Similarly, if the regions with HM are classified as same, then they are considered as TN otherwise FP.

\subsection{Results}

Under the results section, we explore different kinds of results those were obtained after the simulation of proposed mechanism over DIARETDB1 dataset. Table 2 shows the results of first step classification, i.e., the classification of true bright lesions from non-lesions. Here the sensitivity is measured by the fraction of total number of bright lesion regions those were classified correctly divided by the total number of bright lesions regions present in the input retinal images. Next, the specificity is measured as the fraction of total number of nonlesion regions those were classified correctly divided by the total number of non-lesion regions present in the input retinal images. The results are derived for three classifiers such as k-NN, GMM and SVM. Based on these results, the average sensitivity of kNN, GMM and SVM are observed as $79.6304 \%$, $76.5504 \%$ and $88.4774 \%$ respectively. Next, the specificity of k-NN, GMM and SVM are observed as $85.5931 \%, 83.7828 \%$ and $69.0607 \%$ respectively. Furthermore, the maximum specificity is observed at the feature count of 25 and it is approximated as $82.1409 \%$. The maximum sensitivity is observed at the feature count of 30 and it is approximated as $91.7554 \%$. Next, table 3 shows the results of second step classification, i.e., the classification of $\mathrm{HE}$ and CWS. For this case, the maximum sensitivity is observed at 30 feature count and it is approximately $99.1903 \%$ while the average sensitivity of k-NN, GMM and SVM algorithms are observed as $91.6103 \%, 98.0026 \%$ and $97.3525 \%$. Similarly, the maximum specificity is observed at 30 feature count and it is approximately $97.7512 \%$ while the average specificity of k-NN, GMM and SVM algorithms are

Table 2. Bright lesions and non-lesions classification in step 1

\begin{tabular}{|c|c|c|c|c|c|c|}
\hline \multirow{2}{*}{ Features Count } & \multicolumn{3}{|c|}{ Sensitivity (\%) } & \multicolumn{3}{c|}{ Specificity (\%) } \\
\cline { 2 - 7 } & k-NN & GMM & SVM & k-NN & GMM & SVM \\
\hline 5 & 67.1245 & 62.4578 & 67.1452 & 85.1278 & 82.3145 & 63.4578 \\
\hline 10 & 76.1124 & 66.9545 & 89.4785 & 85.3963 & 82.4678 & 62.3679 \\
\hline 15 & 81.4571 & 73.4458 & 84.6696 & 85.5647 & 83.6945 & 69.7845 \\
\hline 20 & 81.5245 & 79.6845 & 95.4578 & 85.7894 & 83.6678 & 76.8947 \\
\hline 25 & 84.5678 & 87.1245 & 95.4785 & 85.3658 & 84.6874 & 76.3696 \\
\hline 30 & 86.9963 & 89.6354 & 98.6345 & 86.3145 & 85.8648 & 65.4897 \\
\hline
\end{tabular}

Table 3. HE and CWS classification in step 2

\begin{tabular}{|c|c|c|c|c|c|c|}
\hline \multirow{2}{*}{ Features Count } & \multicolumn{3}{|c|}{ Sensitivity } & \multicolumn{3}{c|}{ Specificity } \\
\cline { 2 - 7 } & k-NN & GMM & SVM & k-NN & GMM & SVM \\
\hline 5 & 82.6345 & 94.5781 & 95.4678 & 95.4558 & 93.3647 & 98.4596 \\
\hline 10 & 87.4578 & 97.9797 & 97.3658 & 95.4785 & 93.3689 & 96.3458 \\
\hline 15 & 88.9645 & 98.5896 & 95.4878 & 96.2354 & 94.7845 & 99.4568 \\
\hline 20 & 95.6478 & 98.6347 & 97.6389 & 96.3696 & 94.6897 & 99.7548 \\
\hline 25 & 96.3225 & 98.7548 & 98.6969 & 97.0025 & 95.4678 & 99.3658 \\
\hline 30 & 98.6347 & 99.4785 & 99.4578 & 97.5647 & 95.6931 & 99.9958 \\
\hline
\end{tabular}


Table 4. Red lesions and non-lesions classification in step 1

\begin{tabular}{|c|c|c|c|c|c|c|}
\hline \multirow{2}{*}{ Features Count } & \multicolumn{3}{|c|}{ Sensitivity (\%) } & \multicolumn{3}{c|}{ Specificity (\%) } \\
\cline { 2 - 7 } & k-NN & GMM & SVM & k-NN & GMM & SVM \\
\hline 5 & 60.3215 & 61.4578 & 60.2358 & 84.1245 & 85.6336 & 84.7547 \\
\hline 10 & 65.3244 & 64.2385 & 53.2658 & 83.6954 & 85.3696 & 89.6358 \\
\hline 15 & 69.3548 & 69.3645 & 47.2548 & 85.4578 & 84.7525 & 91.4589 \\
\hline 20 & 74.1245 & 70.3251 & 48.5278 & 85.6639 & 85.3482 & 91.6968 \\
\hline 25 & 75.4875 & 72.3654 & 50.1247 & 84.1278 & 83.1247 & 91.3697 \\
\hline 30 & 77.2564 & 74.5689 & 61.8965 & 80.2999 & 85.6947 & 99.4996 \\
\hline
\end{tabular}

Table 5. HMs and MAs classification in step 2

\begin{tabular}{|c|c|c|c|c|c|c|}
\hline \multirow{2}{*}{ Features Count } & \multicolumn{3}{|c|}{ Sensitivity } & \multicolumn{3}{c|}{ Specificity } \\
\cline { 2 - 7 } & k-NN & GMM & SVM & k-NN & GMM & SVM \\
\hline 5 & 75.4214 & 74.1542 & 83.5642 & 90.4521 & 90.2385 & 94.1478 \\
\hline 10 & 76.3258 & 75.6895 & 86.3457 & 90.5685 & 90.8964 & 95.6378 \\
\hline 15 & 84.5689 & 79.3658 & 87.9645 & 90.6986 & 91.4585 & 96.8574 \\
\hline 20 & 93.6584 & 80.2355 & 85.9647 & 91.7548 & 91.3696 & 97.6986 \\
\hline 25 & 94.3685 & 81.4665 & 94.5638 & 91.6638 & 92.8967 & 97.9685 \\
\hline 30 & 95.8654 & 82.6354 & 97.4127 & 92.4596 & 90.2585 & 99.4785 \\
\hline
\end{tabular}

observed as $96.3511 \%, 94.5614 \%$ and $98.8964 \%$.

Table 4 shows the results of first step classification of true red lesions and non-red lesions. In this case, the sensitivity is measured as the ratio of total number of correctly classified red-lesion regions to the total number of red-lesion regions present in the input image. The denominator value is obtained through ground truth image and numerator is obtained through the proposed classification method. Similarly, the specificity is measured as the ratio of correctly classified non-red-lesion regions to the total number of non-red-lesion regions present in the input image. The obtained sensitivity and specificity at the first classification step are shown in Table 4. According to the specified results, the maximum sensitivity is gained for 30 feature count at the k-NN classifier. The average sensitivity of k-NN, GMM and SVM are observed as $70.3115 \%, 68.7200 \%$ and $53.5509 \%$ respectively. Moreover, the average sensitivity at features $5,10,15,20,25$, and 30 is observed as $60.6777 \%, 60.9429 \%, 61.9914 \%$, $64.3258 \%, 65.9925 \%$ and $71.2406 \%$ respectively. The maximum specificity is gained for 30 feature count at the k-NN classifier. The average specificity of k-NN, GMM and SVM are observed as $83.8949 \%$, $84.9872 \%$ and $91.4026 \%$ respectively. Moreover, the average sensitivity at features $5,10,15,20,25$, and 30 is observed as $84.8376 \%, 86.2336 \%$, $87.2231 \%, 87.5696 \%, 86.2074 \%$ and $88.4981 \%$ respectively. Based on these results, we can understand that the k-NN classifier had shown better performance compared to the remaining algorithms. The results derived at the second step classification of red-lesions are shown in table 5. At this classification phase, the red-lesions are classified into two sub-classes such as MA and HM. For this classification, the maximum sensitivity is observed at SVM classifier and it is approximately $89.3026 \%$ while for the remaining classifiers, it is observed as $86.7014 \%$ and $78.9245 \%$ for k-NN and GMM respectively. Based on these results, the SVM is declared as a best classifier. The difference between lesion and non-lesion regions is very much high and hence the simple k-NN classifier had gained better performance. For sub-classes, the provision of feature discrimination is very important and then only they can be differentiated effectively. Hence the SVM had gained a better performance at the second step classification.

Fig. 5 shows the accuracy details evaluated at the classification lesions (both bright and red) through different classifiers. Here, the accuracy is derived by

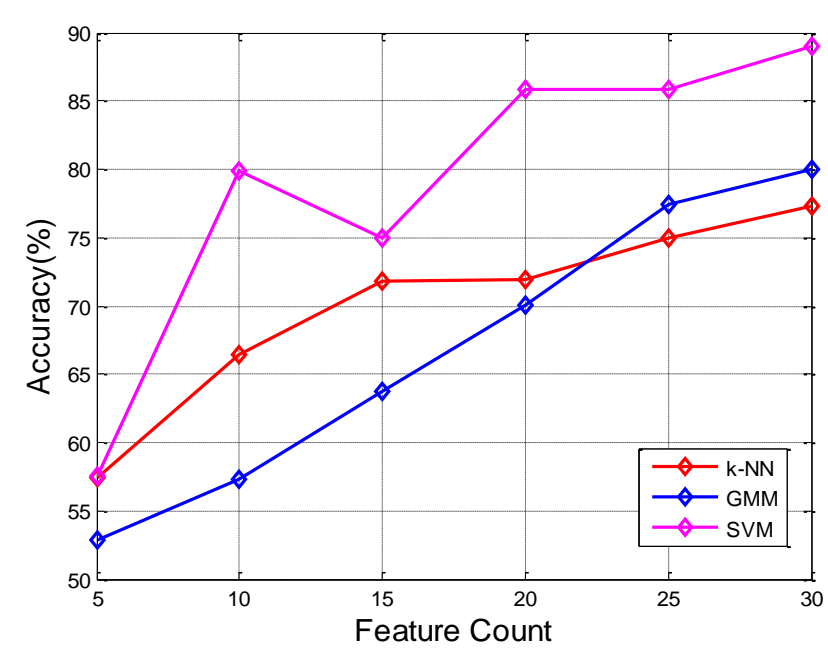

Figure. 5 Accuracy (\%) for varying feature count at different classifiers 
Table 6. Comparison with existing methods

\begin{tabular}{|c|c|c|c|c|}
\hline Author & Methodology & Dataset used & Method & Performance \\
\hline Pratt et al. [33] & 13 layered CNN & DIARETDB1 & DR grading & $\begin{array}{c}\text { Sen }-91.2, \\
\text { Acc }-75 \%\end{array}$ \\
\hline M. K. Fadafen [13] & $\begin{array}{c}\text { Mathematical } \\
\text { Morphology }\end{array}$ & DIARETDB1 & Exudates Segmentation & AUC - 90.12\% \\
\hline Proposed & $\begin{array}{c}\text { Morphology, Filtering, } \\
\text { Composite features, k- } \\
\text { NN, GMM and SVM }\end{array}$ & DIARETDB1 & $\begin{array}{c}\text { HM, EX, CWS, MA } \\
\text { classification followed by } \\
\text { DR grading }\end{array}$ & $\begin{array}{l}\text { Sen }-95.2 \%, \text { Spe }- \\
82.1 \%, \text { Acc }-90.2 \% \\
\text { and AUC }-88.5 \%\end{array}$ \\
\hline
\end{tabular}

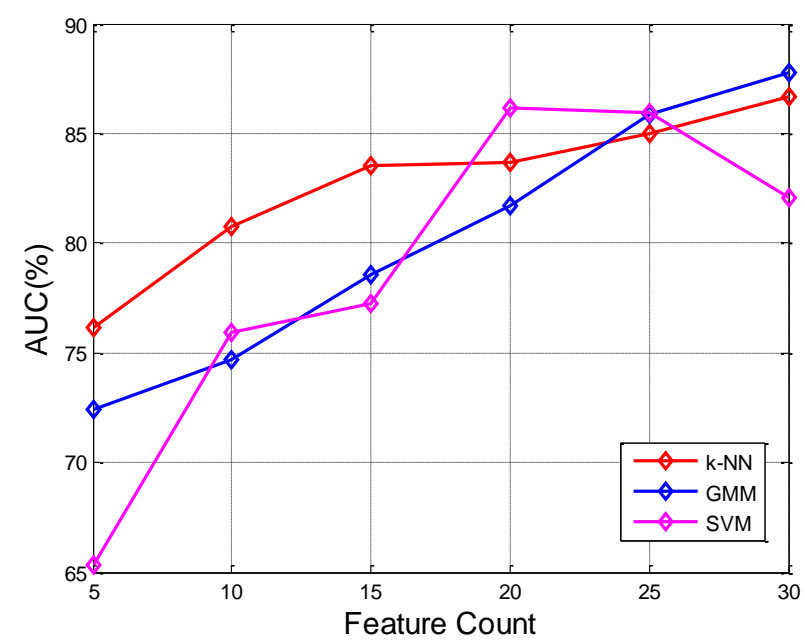

Figure. 6 AUC (\%) for varying feature count at different classifiers

averaging the accuracies obtained at the classification of bright and red lesions. With an increase in feature count, the accuracy is increasing because, as the feature number is increased, the classifier will get a full clarity over the regions and it can classify them effectively. Among the three algorithms, the maximum accuracy is obtained by the SVM because it can differentiate even the closer regions much effectively. On the other hand, the 30 features are most relevant and much discriminative features which provides add on boost to classifier. The next metric, AUC is nothing but an average of sensitivity and specificity. This is calculated as an average of AUCs of red and bright lesions. Figure.6 shows the AUC details evaluated at the classification lesions (both bright and red) through different classifiers. The average AUC of k-NN, GMM and SVM is observed as $82.6118 \%, 80.1666 \%$ and $78.7690 \%$ respectively. Based on these values, we can demonstrate that the simple k-NN classifier has gained an excellent performance in the classification of both lesion regions and non-lesion regions.

Table 6 shows the comparison between proposed and existing method through methodology, method, performance and datasets used for validation. M. K. Fadafen [13] used Mathematical morphology which is the basic version of segmentation methodology.
Even though they have employed machine learning algorithms for classification, they gained a better performance at segmentation level but a less concentrate was made on the DR grading. Moreover, they focused on the pixel level classification which declares the difference between segmented region through the applied methodology and ground truth. This kind of analysis won't give any idea about DR grading and most of them stopped at the classification phase only. Even though our method has gained less AUC compared to [13], it was a full-pledged system that ends up with severity grading. Next, Pratt et al. [33] employed a 13-layered CNN model for the grading of DR. However, they didn't concentrate on the quality of image, segmentation and lesions identification. They applied the CNN model directly over retinal images which introduce so many problems at severity grading. Hence it obtained only $75 \%$ accuracy which is very low compared to the accuracy of proposed system.

\section{Conclusion}

In this paper, we have proposed a four stage automatic DR screening system that identifies and grade's the stage of DR based on retinal fundus images. Since the OD and Blood vasculature are the major parts of retinal images, initially they are subjected to segmentation after pre-processing the image. In the next phase, the residual image (lesion image) is subjected to feature extraction followed by a hierarchical classification that classifies the entire regions in to four classes. The first phase classifications dedicated for the separation of lesion regions from non-lesion regions and the second phase classification concentrated on the sub-class classification. Finally, the entire regions of retinal images are classified as MA, HM, HE and CWS which are further used for the grading of DR severity. For experimental validation, we used DIARETDB1 dataset with three different machine learning algorithms such as k-NN, GMM and SVM. The maximum accuracy $(86.6521 \%)$ of first phase classifications is observed at 30 features with $\mathrm{k}-\mathrm{NN}$ classifier while the maximum accuracy $(99.6345 \%)$ 
of second phase classifications is observed at 30 features with SVM classifier.

This paper developed a complete DR diagnosis system which considered all the possible attributes present in retinal images. The developed also gained a maximum accuracy that proves the efficiency. However, it was tested only on limited retinal images. In future, to check the robustness, it can be applied over different retinal images with different DR statuses.

\section{References}

[1] T. Y. Wong and C. Sabanayagam, "Strategies to tackle the global burden of diabetic retinopathy: From epidemiology to artificial intelligence", Ophthalmologica, Vol. 243, No. 1, pp. 9-20, 2020.

[2] N. Congdon, Y. Zheng, and M. He, "The worldwide epidemic of diabetic retinopathy", Indian Journal of Ophthalmology, Vol. 60, No. 5, pp. 428-431, 2012.

[3] I. N. Figueiredo, S. Kumar, C. M. Oliveira, J. D. Ramos, and B. Engquist, "Automated lesion detectors in retinal fundus images", Computers in Biology and Medicine, Vol. 66, No.1, pp. 4765, 2015.

[4] M. D. Abràmoff, M. Niemeijer, M. S. A. S. Schulten, M. A. Viergever, S. R. Russell, B. V. Ginneken, "Evaluation of a system for automatic detection of diabetic retinopathy from color fundus photographs in a large population of patients with diabetes", Diabetes Care, Vol. 31, No. 2, pp. 62-83, 2008.

[5] T. Hassan, M. U. Akram, B. Hassan, A. Nasim, and S. A. Bazaz, "Review of OCT and fundus images for detection of macular edema", In: Proc. of IEEE International Conf. on Imaging Systems and Techniques, Macau, China, pp. 1-4, 2015.

[6] A. Biran, P. S. Bidari, and K. Raahemifar, "Automatic method for exudates and hemorrhages detection from fundus retinal images", International Journal of Computer Information Engineering, Vol. 10, No. 9, pp. 1599-1602, 2016.

[7] A. M. Reza, "Realization of the contrast limited adaptive histogram equalization (CLAHE) for real-time image enhancement," Journal of VLSI Signal Processing Systems for Signal, Image and Video Technology, Vol. 38, No. 1, pp. 3544, 2004.

[8] G. L. Atlas and K. Parasuraman, "Detection of retinal hemorrhage from fundus images using ANFIS classifier and MRG segmentation",
Biomedical Research, Vol. 29, No. 7, pp. 14891497, 2018.

[9] X. Tang, "Texture information in run-length matrices", IEEE Transactions on Image Processing, Vol. 7, No. 11, pp. 1602-1609, 1998.

[10] N. Loris, B. Sheryl, G. Stefano, M. Emanuele, B. Tonya, "Different Approaches for Extracting Information from the Co-Occurrence Matrix", PLOS ONE, Vol. 8, No. 12, p. e83554, 2013.

[11] H. Bay, E. Andreas, T. Tuytelaars, and L. V. Gool, "SURF: Speeded Up Robust Features", Computer Vision and Image Understanding (CVIU), Vol. 110, No. 3, pp. 346-359, 2008.

[12] J. I. Orlando, E. Prokofyeva, and M. B. Blaschko, "A discriminatively trained fully connected conditional random field model for blood vessel segmentation in fundus images", IEEE Transactions on Biomedical Engineering, Vol. 64, No. 1, pp. 16-27, 2017.

[13] M. K. Fadafen, N. Mehrshad, and S. M. Razavi, "Detection of diabetic retinopathy using computational model of human visual system", Biomedical Research, Vol. 29, No. 9, pp. 19561960, 2018.

[14] D. W. Safitri and D. Juniati, "Classification of diabetic retinopathy using fractal dimension analysis of eye fundus image", In: Proc. of International Conf. on Mathematics: Pure, Applied and Computation, Surabaya, Indonesia, pp. 020011-1-11, 2017.

[15] J. Z. Liu, L. D. Zhang, and G. H. Yue, "Fractal Dimension in Human Cerebellum Measured by Magnetic Resonance Imaging", Biophysical Journal, Vol. 85, No. 6, pp. 4041-4046, 2003.

[16] E. A. Maksoud, S. Barakat, and M. Elmogy, "A comprehensive diagnosis system for early signs and different diabetic retinopathy grades using fundus retinal images based on pathological changes detection", Computers in Biology and Medicine, Vol. 126, No. 104039, pp. 512-542, 2020.

[17] M. D. Abràmoff, Y. Lou, A. Erginay, W. Clarida, R. Amelon, J. C. Folk, and M. Niemeijer, "Improved automated detection of diabetic retinopathy on a publicly available dataset through integration of deep learning", Investigative Ophthalmology and Visual Science, Vol. 57, No. 13, pp. 5200-5206, 2016.

[18] M. Savoy, "IDx-DR for Diabetic Retinopathy Screening", Am Fam Physician, Vol. 101, No. 5, pp. 307-308, 2020.

[19] C. Kumar, S. Puri, and P. Simard "High Performance Convolutional Neural Networks for Document Processing", In: Proc. of Tenth International Workshop on Frontiers in 
Handwriting Recognition, La Baule, France, pp. 1-7, 2006.

[20] V. Bellemo, Z. W. Lim, G. Lim, Q. D. Nguyen, Y. Xie, M. Y. Yip, H. Hamzah, J. Ho, X. Q. Lee, W. Hsu, M. L. Lee, L. Musonda, M. Chandran, G. C. Mutati, M. Muma, G. S. W. Tan, S. Sivaprasad, G. Menon, T. Y. Wong, and D. S. W. Ting, "Artificial intelligence using deep learning to screen for referable and visionthreatening diabetic retinopathy in africa: A clinical validation study", Lancet Digital Health, Vol. 1, No. 1, pp. e35-e44, 2019.

[21] H. Kaiming, X. Zhang, R. Shaoqing, and S. Jian, "Deep Residual Learning for Image Recognition", In: Proc. of International Conf. on Computer Vision and Pattern Recognition, Las Vegas, NA, USA, pp. 770-778, 2015.

[22] K. Simonyan and A. Zisserman, "Very Deep Convolutional Networks for Large-Scale Image Recognition", In: Proc. of International Conf. on learning representations, Vancouver, BC, Canada, pp. 1-14, 2014.

[23] R. F. Mansour, "Deep-learning-based automatic computer-aided diagnosis system for diabetic retinopathy", Biomedical Engineering Letters, Vol. 8, No. 1, pp. 41-57, 2018.

[24] [24] D. G. Bailey, C. T. Johnston, N. Ma, "Connected components analysis of streamed images", In: Proc. of International Conf. on Field Programmable Logic and Applications, Heidelberg, pp. 679-682, 2008.

[25] T. R. Gadekallu, N. Khare, S. Bhattacharya, S. Singh, P. K. R. Maddikunta, I. H. Ra, and M. Alazab, "Early detection of diabetic retinopathy using PCA-firefly based deep learning model", Electronics, Vol. 9, No. 2, pp. 1-16, 2020.

[26] H. Zou and L. Xue, "A Selective Overview of Sparse Principal Component Analysis”, In: Proc. of the IEEE, Vol.106, No. 8, pp. 1311-1320, 2018.

[27] M. T. Hagos and S. Kant, "Transfer learning based detection of diabetic retinopathy from small dataset", arXiv:1905.07203, 2019.

[28] M. Barker and Adam, "Inception V3 Deep Convolutional Architecture for Classifying Acute Myeloid / Lymphoblastic Leukemia", intel.com. Retrieved on November 2021.

[29] B. Tymchenko, P. Marchenko, and D. Spodarets, "Deep learning approach to diabetic retinopathy detection", arXiv:2003.02261, 2020.

[30] K. Alex, S. Ilya, and H. E. Geoffrey, "ImageNet classification with deep convolutional neural networks", Communications of the ACM., Vol. 60, No. 6, pp. 84-90, 2017.
[31] S. Mukund and N. Amir, "The many Shapley values for model explanation", In: Proc. of International Conf. on Machine Learning, PMLR, Vol. 119, pp.9269-9278, 2020.

[32] K. Xu, D. Feng, and H. Mi, "Deep convolutional neural network-based early automated detection of diabetic retinopathy using fundus image", Molecules, Vol. 22, No. 12, p. 2054, 2017.

[33] H. Pratt, F. Coenen, D. M. Broadbent, S. P. Harding, and Y. Zheng, "Convolutional neural networks for diabetic retinopathy", Procedia Computer Science, Vol. 90, pp. 200-205, 2016.

[34] Y. M. S. Reddy, R. S. E. Ravindran, and K. H. Kishore, "Spatial Mutual Relationship Based Retinal Image Contrast Enhancement for Efficient Diagnosis of Diabetic Retinopathy", International journal of Intelligent Engineering Systems, Vol. 11, No. 4, pp. 48-58, 2018.

[35] Y. M. S. Reddy and R. S. E. Ravindran, "A Composite Feature Set Based Blood Vessel Segmentation in Retinal Images through Supervised Learning", JMCMS, Vol. 14, No. 5, pp. 111-130, October, 2019.

[36] A. F. Frangi, W. J. Niessen, K. L. Vincken, and M. A. Viergever, "Multiscale vessel enhancement filtering", In: Proc. of International Conference on Medical Image Computing and Computer-Assisted Intervention, Springer, Berlin Heidelberg, pp. 130-137, 1998.

[37] Y. M. S. Reddy and R. S. E. Ravindran, "Optic Disk Segmentation through Edge Density Filter in Retinal Images", International Journal of Innovative Technology and Exploring Engineering, Vol. 9, No. 3, pp. 3168-3176, 2020.

[38] N. Otsu, "A threshold selection method from gray-scale histogram", IEEE Transactions on Systems Man Cybernetics, Vol. 8, No.1, pp. 6266, 1978

[39] A. Frame, P. Undrill, M. Cree, J. Olson, K. McHardy, P. Sharp, and J. Forrester, "A comparison of computer based classification methods applied to the detection of microaneurysms in ophthalmic fluorescein angiograms", Computers in Biology and Medicine, Vol. 28, No. 3, pp. 225-238, 1998.

[40] M. Niemeijer, B. van Ginneken, J. Staal, M. Suttorp Schulten, and M. Abramoff, "Automatic detection of red lesions in digital color fundus photographs", IEEE Transactions on Medical Imaging, Vol. 24, No. 5, pp. 584 -592, May, 2005.

[41] M. G. Lawrence, "The accuracy of digital-video retinal imaging to screen for diabetic retinopathy: an analysis of two digital-video retinal imaging systems using standard 
Received: November 19, 2021. Revised: December 28, 2021.

stereoscopic seven-field photography and dilated clinical examination as reference standards", Trans Am Ophthalmol Soc, Vol. 102, pp. 321-340, 2004. 